\title{
Molecular Detection of vanA and vanB Genes in Vancomycin-Resistant Enterococcus Isolated by Polymerase Chain Reaction From the Intestines of Children Admitted to the Intensive Care Units
}

\author{
Zahra Daghighi ${ }^{1}$; Saeed Tajbakhsh ${ }^{1,2}$; Hossein Goudarzi ${ }^{3}$; Abdollah Karimi ${ }^{4}$; Alireza \\ Nateghian $^{5, *}$ \\ ${ }^{1}$ Department of Microbiology and Parasitology, Faculty of Medicine, Bushehr University of Medical Sciences, Bushehr, IR Iran \\ ${ }^{2}$ The Persian Gulf Tropical Medicine Research Center, Bushehr University of Medical Sciences, Bushehr, IR Iran \\ ${ }^{3}$ Department of Microbiology, Shahid Beheshti University of Medical Sciences, Tehran, IR Iran \\ ${ }_{5}^{4}$ Pediatric Infections Research Center, Mofid Children's Hospital, Shahid Beheshti University of Medical Sciences, Tehran, IR Iran \\ ${ }^{5}$ Ali-Asghar Children's Hospital, Iran University of Medical Sciences, Tehran, IR Iran \\ ${ }^{*}$ Corresponding author: Alireza Nateghian, Ali-Asghar Children's Hospital, Iran University of Medical Sciences, Tehran, IR Iran. Tel: +98-2122858143, Fax: +98-2122220063, E-mail: nat- \\ eghian@hotmail.com; anateghian@tums.ac.ir
}

Received: February 22,2014; Revised: March 24, 2014; Accepted: April 27, 2014

\begin{abstract}
Background: Enterococci are considered as the third most common cause of nosocomial infections and their antimicrobial resistance has been a concerning issue.

Objectives: In this study, we looked for resistance genes of vanA and vanB in vancomycin-resistant Enterococcus (VRE) isolated from intestinal colonization of children admitted to the pediatric intensive care unit (PICU) and neonatal ICU (NICU) of Ali-Asghar Children's Hospital.

Patients and Methods: In this descriptive study, 71 rectal swab samples were collected from the intestines of children admitted to the PICU and NICU of Ali-Asghar Children's Hospital. Enterococci were diagnosed in samples by appropriate microbiological tests. Antimicrobial resistance and VRE detection was performed by the Kirby-Bauer disk diffusion method on Mueller-Hinton agar based on Clinical and Laboratory Standards Institute (CLSI) criteria. vanA and vanB genes were detected by PCR.

Results: Enterococcus was detected in 64 (90.1\%) rectal swab samples. The frequency rate of VRE strains was 47 (73.4\%) and vancomycinintermediate Enterococcus (VIE) strains was 6 (9.4\%). PCR analysis of VRE samples showed that 42 samples had vanA gene (89.3\%) but vanB gene was not identified in remaining five samples. VIR was detected in 4 cases with vanA gene (66.7\%). Again, we did not $\mathrm{d}$ vanB gene in remaining samples.

Conclusions:VRE colonization was very high among studied cases. Most important mechanism for high level of resistance to vancomycin is presence of van genes, which can be potentially transmittable to other enterococci and gram-positive organisms. More molecular studies are needed to clarify the trend of VRE colonization and the role of preventive measures in this setting.
\end{abstract}

Keywords:Enterococcus; Vancomycin; Polymerase Chain Reaction; vanB protein

\section{Background}

Enterococci can be considered as gastrointestinal and vaginal flora (1). However, these bacteria are also considered as the third most common cause of nosocomial infections, which are now spreading and are hard to treat around the world included Iran (2). Enterococci can invade bloodstream and can cause urinary tract infection, endocarditis, peritonitis, and many other types of infections $(2,3)$. Antimicrobial resistance of these organisms has been a concerning issue for a long time (1-4). These organisms are inherently resistant to cephalosporins, clindamycin, and many other antibiotics. Beta-lactamase production by some strains of Enterococcus faecalis and Enterococcus faecium is also another mechanism of resis- tance. Vancomycin-resistant Enterococcus (VRE) not only show high-grade resistance to vancomycin and aminoglycosides, but also may show resistance to penicillins (3).

Resistance to vancomycin in these organisms is an important problem because this antibiotic is very effective against gram-positive bacteria whose rate of resistance has been increasing during recent years due to increase in the presence of plasmid bearing resistance genes (5). The resistance inducing gene for vancomycin is called van gene and is subtyped to type $A$ and $B$ on transposon Tn1546. These genes can be potentially introduced to conjugative plasmid, transferred within enterococcal strains 
as well as to the other organisms such as staphylococci, and can increase the potential risk of vancomycin-resistant Staphylococcus (VRS) in the community. These two genes cause high-grade resistance to vancomycin while genes type $D, C$, and $E$ cause low-grade resistance to vancomycin and are located on chromosome $(6,7)$. Virulence factors specific to glycopeptide-resistant Enterococcus have not been identified so far (5). It seems that their virulence is similar to vancomycin-sensitive strains and although the mortality rate is higher, it can be attributed to failure of on time treatment. Strains positive for $v a n A$ are usually resistant to both vancomycin and teicoplanin but vanB positive strains usually respond to the later one (5). Therefore, determination of these resistance genes is important for therapeutic strategies.

\section{Objectives}

In this study, we looked for resistance genes of vanA and $\operatorname{vanB}$ in VRE isolated from intestinal colonization of children admitted to the pediatric and neonates intensive care units (PICU and NICU, respectively) of Ali-Asghar Children's Hospital during 2012-2013. The Ethical Committee of Pediatric Infections Research Center of Mofid Children's Hospital approved the study protocol.

\section{Patients and Methods}

\subsection{Bacterial Identification and Patients Selection}

This descriptive study was conducted from January 2012 to June 2013. Surveillance of VRE colonization (rectal or stool swab) was performed on all children aged 18 months or younger admitted to the PICU and NICU of Ali-Asghar Children's Hospital, Tehran, who met the inclusion criteria. The inclusion criteria were serious systemic illness including admission to NICU or PICU for at least one week, malignancy, chronic kidney, lung, or liver diseases, treatment with chemotherapeutic agents, immunodeficiency, treatment with high-dose corticosteroids (more than $1 \mathrm{mg} / \mathrm{kg} /$ day for more than one month), malnutrition (body weight $<5$ th percentile for age), and previous treatment with second or third generation cephalosporins, aminoglycosides, or broad-spectrum $\beta$-lactams within the past three months. We exclude children with proven enterococcal infection and children whose parents were not willing to participate in this study.

Rectal swab samples were transferred to the Pediatric Infections Research Center, Mofid Children's Hospital, immediately after obtaining. Enterococci were diagnosed in samples by Gram staining, biochemical tests like catalase, growing on bile esculin agar and $\mathrm{NaCl}(6.5 \%)$ media, and ability to growth on selective media of Enterococcosel agar.

\subsection{Antimicrobial Susceptibility Testing}

Antimicrobial susceptibility to ampicillin $(10 \mu \mathrm{g})$, peni- cillin $(10 \mu \mathrm{g})$, ciprofloxacin $(5 \mu \mathrm{g})$, rifampin $(5 \mu \mathrm{g})$, teicoplanin $(30 \mu \mathrm{g})$, chloramphenicol $(30 \mu \mathrm{g})$, vancomycin $(30 \mu \mathrm{g})$, quinupristin $(15 \mu \mathrm{g})$, and linezolid $(30 \mu \mathrm{g})$ (Mast Group, Merseyside, UK) was assessed by the Kirby-Bauer disk diffusion method on Mueller-Hinton agar (Merck, Germany) based on Clinical Laboratory Standards Institute (CLSI) criteria (8). Enterococcus faecalis ATCC 29212 was used as the control strain (8).

According to CLSI criteria, vancomycin-sensitive Enterococcus (VSE) is defined as inhibition zone equal or greater than $17 \mathrm{~mm}$. In addition, vancomycin-intermediate Enterococcus (VIE) and VRE are defined as the inhibition zone of 15 to $16 \mathrm{~mm}$ and equal or less than $14 \mathrm{~mm}$, respectively.

\subsection{DNA Extraction}

Total DNA of the different bacterial isolates was extracted by the DNA extraction kit (Cat. No. K-3032-2, Bioneer Company, Korea). The process was performed as follows:

Two hundreds of phosphate buffer was added to the microtubes and colonies of bacteria were solved in microtubes; proteinase $\mathrm{K}(20 \mathrm{~mL})$ and binding buffer $(200 \mathrm{~mL})$ were added to the microtubes. Then microtubes were placed in a water bath for ten minutes and elution buffer was placed in water bath at the same time. Thereafter, isopropanol $(100 \mathrm{ml})$ was added to the microtubes. Microtubes content were transferred to the filtered microtubes and they were centrifuged. Microtubes content were emptied and washing buffer $1(500 \mathrm{~mL})$ was added and they were centrifuged. Microtubes content were emptied again and washing buffer $2(500 \mathrm{~mL})$ was added and they were centrifuged. Microtubes content were empty once more and they were centrifuged. Then elution buffer (100 $\mathrm{mL}$ ) was added to the microtubes and they were centrifuged. Extracted DNA samples were transferred to new microtubes.

\subsection{Detection of Virulence Genes by Polymerase Chain Reaction}

Polymerase chain reaction (PCR) method was performed on enterococcal isolates for detection of $v a n A$ and vanB genes. Used primers are presented in Table 1. Briefly, the $25 \mu \mathrm{L}$ of PCR mixture contained $2.5 \mu \mathrm{L}$ of bacterial DNA, $10 \mathrm{pM}$ of each primers, $1.5 \mathrm{mM}$ of $\mathrm{MgCl}_{2}, 250 \mu \mathrm{M}$ of each dNTP, 10 mM of Tris-HCL ( $\mathrm{pH}=9.0$ ), $30 \mathrm{mM}$ of KCL, and $1 \mathrm{U}$ of Taq DNA polymerase (Bioneer Company-Korea, Cat. No. K-2012). Reactions were performed on thermal cycler (Eppendorf, Master cycler gradient). Amplification for vanA was performed with the following thermal cycling conditions: ten minutes at $95^{\circ} \mathrm{C}$ and 30 cycles of amplification consisting of one minute at $94^{\circ} \mathrm{C}$, one minute at $57^{\circ} \mathrm{C}$, one minute at $72^{\circ} \mathrm{C}$, and ten minutes at $72^{\circ} \mathrm{C}$ for the final extension. Amplification for vanB was performed with the following thermal cycling conditions: 10 minutes at $95^{\circ} \mathrm{C}$ and 30 cycles of amplification consisting of one minute at $94^{\circ} \mathrm{C}$, one minute at $60^{\circ} \mathrm{C}$, one minute at $72^{\circ} \mathrm{C}$, and ten 


\begin{tabular}{|c|c|c|}
\hline Target & Product Length, bp & Company \\
\hline $\operatorname{van} A$ & 1030 & \\
\hline vanA-F: 5'CATGAATAGAATAAAAGTTGCAATA3' & & Bioneer \\
\hline vanA-R: 5'CCCCTTTAACGCTAATACGATCAA3' & & Bioneer \\
\hline vanB & 433 & \\
\hline vanB-F: 5'GTGACAAACCGGAGGCGAGGA3' & & Bioneer \\
\hline vanB-R: 5'CCGCCATCCTCCTGCAAAAAA3' & & Bioneer \\
\hline
\end{tabular}

Table 2. Demographic and Clinical Characteristics of Patients $(n=64)$ a, b

\begin{tabular}{|c|c|c|c|c|}
\hline Characteristic & Positive for Growth of Enterococcus & $\operatorname{VIE}(\mathbf{n}=6)$ & $\operatorname{VSE}(\mathbf{n}=\mathbf{1 1})$ & $\operatorname{VRE}(n=47)$ \\
\hline Solid tumor & $3(4.2)$ & - & - & $3(4.2)$ \\
\hline Diabetes mellitus & $2(2.8)$ & - & $1(1.4)$ & $1(1.4)$ \\
\hline Chronic renal disease & $4(5.6)$ & $1(1.4)$ & $2(2.8)$ & $1(1.4)$ \\
\hline Treatment with chemotherapeutic agents & $8(11.3)$ & - & - & $8(11.3)$ \\
\hline Blood dyscrasia & $8(11.3)$ & - & - & $8(11.3)$ \\
\hline Chronic lung disease & $3(4.2)$ & - & $1(1.4)$ & $2(2.8)$ \\
\hline Immunodeficiency & $3(4.2)$ & - & - & $3(4.2)$ \\
\hline Treatment with corticosteroids & $2(2.8)$ & - & - & $2(2.8)$ \\
\hline Presence of invasive device & $44(62)$ & $5(7)$ & $8(11.3)$ & $31(43.7)$ \\
\hline Previous ICU admission in the past 3 months & $11(15.5)$ & - & $2(2.8)$ & $9(12.7)$ \\
\hline previous treatment with antibiotics & $12(16.9)$ & $2(2.8)$ & $1(1.4)$ & $9(12.7)$ \\
\hline ICU admission over 7 days & $47(66.2)$ & $4(5.6)$ & $7(9.9)$ & $36(50.7)$ \\
\hline
\end{tabular}

a Abbreviations: VIE, vancomycin-intermediate Enterococcus; VRE, vancomycin-resistant Enterococcus; VSE, vancomycin-sensitive Enterococcus.

b Data are presented as No.(\%).

Figure 1. PCR Amplification of vanA Gene of Enterococci Isolates

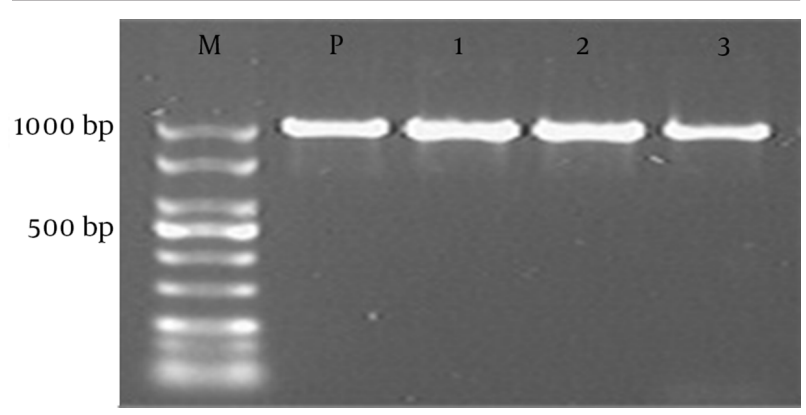

Lane M, DNA size marker; lane P, positive control; lane 1, 2, and 3: vanA (1030 bp) gene positive isolates.

minutes at $72^{\circ} \mathrm{C}$ for the final extension. PCR product bands were analyzed after electrophoresis on a $1 \%$ agarose gel at $100 \mathrm{~V}$ for 60 minutes in $1 \mathrm{X}$ TBE containing ethidium bromide and the result was checked under ultraviolet irradiation.

\subsection{Sequencing Method}

The PCR purification kit (Bioneer Co., Korea) was used to purify PCR products and sequencing was performed by the (Bioneer Company, Korea). The nucleotide sequences were analyzed with the Chromas 1.45 software and BLAST in NCBI.

\section{Results}

Seventy-one patients who met the inclusion criteria over a period of 18 months were enrolled in this study. Amongst them, 38 (53.5\%) were males and 33 (46.5\%) were females; mean age of patients was $29.1 \pm 38.5$ months (ranging from two days to 147.5 months). Sixty-four (90.1\%) patients were colonized with enterococci. Of 64 strains, 47 (73.4\%) were VRE. The remaining isolates were either VSE (11 strains, 17.2\%) or VIE (six strains, 9.4\%). Table 2 shows other demographic and clinical characteristics of the patients. The resistance rate of enterococci isolates to tested antibiotics were $85.11 \%$ to ampicillin, $80.85 \%$ to ciprofloxacin, $82.98 \%$ to penicillin, $87.23 \%$ to rifampin, $78.72 \%$ to teicoplanin, $46.81 \%$ to chloramphenicol, $23.40 \%$ to quinupristin and $2.13 \%$ to linezolid. PCR analysis of VRE samples showed that $42(89.3 \%)$ samples had vanA gene but vanB gene was not detected in remaining samples. In VIE group, vanA gene was also detected in 4 (66.7\%) cases. 
Again, we did not detect vanB gene in remaining samples (Figure 1).

\section{Discussion}

This study showed that vanA gene was the dominant resistance mechanism in isolated enterococcal colonization from patients admitted to ICU of this pediatric tertiary educational center in Tehran, Iran, during 2012-2013. Although many risk factors have been proposed to affect VRE colonization, assessment of these risk factors was not our primary goal in this study due to our small sample size. We used previous proposed risk factors to collect as much as possible VRE strain to look for resistance genes. However, it should be noted the among some of inclusion criteria, ICU admission over seven days, previous ICU admission in the past three months, presence of invasive devices, treatment with previous antibiotic, treatment with chemotherapeutic agents and having underlying hematologic problems seems to put patients more at risk of colonization $(5,9)$.

VRE colonization rates were investigated in various settings with inconsistent results; in a study in a tertiary care center in Australia, univariate analysis showed that the use of any antibiotic including meropenem as well as ciprofloxacin, diarrhea, and longer length of hospital stay were associated with increased risk of VRE colonization. The predominant VRE genotype circulating in Australia is E. faecium vanB. In contrast, the vanA gene was predominant one in our study; however, our result was more compatible with VRE genotype status in United States and Europe (9). In another study in Italia, it was shown that 20 out of 26 VRE isolates from patients admitted to an ICU had vanA gene (10) while remainder showed vanB gene; a finding that was different from our results. Although remainder of our non-vanA VRE isolates might have less resistant genotypes, such a hypothesis must be investigated in future studies. Increasing enterococci with vanA gene rate in a hospital is a concerning issue for whole country. A study in Germany investigated the samples of different origin in various hospitals over the country between 2004-2006 and analyzed them by multilocus sequence typing (MLST), SmaI macrorestriction analysis in pulsed-field gel electrophoresis (PFGE), and multiplelocus variable-number tandem repeat analysis (MLVA) (11). A dissemination of related vancomycin-resistant $E$. faecium among various hospitals and Federal States was proved by spreading an identical vanA gene clusters among clonally different strain types. Hence, adherence to infection control measures especially in ICU settings is very important (11).

Our previous study in 2008 in Ali-Asghar Children Hospital, which was focused on VRE rectal colonization rate, detected VRE in stools from 33 (25\%) of 130 children with acute lymphoblastic leukemia (ALL). No clear risk factors were identified for VRE colonization in that study, but there was a trend towards an increased prevalence in children admitted to the ICU since their ALL diagnosis $(\mathrm{P}=0.07)$. The vanA gene was found in $28(85 \%)$ of the 33 stools, with all other enterococci being $\operatorname{vanB}(12)$.

Although we assessed the rectal colonization in selected high-risk patients, the observed high rate in our study is concerning. Most studies in developed countries showed lower rates of colonization. In a study in Australia in a general hospital, VRE was detected from patients in each ward with the prevalence ranging from $3 \%$ to $29 \%$ and concluded that exposure to some antibiotics, especially meropenem, might explain the increasing rate of colonization. Therefore, the antimicrobial prescription in our setting should be closely monitored to prove their role in this high and increasing rate of colonization (9). Present study confirmed that ICU admission in our setting might be considered as a risk factor for VRE colonization and the fact that rate of colonization in selected cases was much higher in comparison with previous study. Moreover, the genotypes could be changed over time, as we did not detect any vanB gene in present study even in patients with malignancies. This finding necessitates further molecular studies to clarify the exact nature of resistant genes in these isolates.

Our study has some limitations; the sample size for most potentially risk factors was too low to do statistical analysis; hence, a multicentric national study is suggested to investigate the trend and exact risk factors in each hospital setting that would lead to design scientific preventive measures.

\section{Acknowledgements}

This article was the postgraduate thesis for Master of Science degree of Zahra Daghighi. We would like to thank the personnel of Pediatric Infections Research Center, Dr Masoumeh Navidinia, Mrs. Zari Gholinejad, Microbiology department of Shahid Beheshti University of Medical Sciences, and Mrs. Masoumeh Miradi, the infection control nurse of Ali-Asghar Children's Hospital for their collaboration in this study. It shall be noted that this research could not be done without support of Dr Abdollah Karimi, the president of Pediatric Infections Research Center.

\section{Authors' Contribution}

Dr. Saeed Tajbakhsh, Hossein Goudarzi, and Abdollah Karimi had guidance for proposal and Zahra Daghighi did the test; Dr. Nateghian provided the samples; Abdollah Karimi 4; Alireza Nateghian wrote the paper. The study was a thesis for Master of Science degree in microbiology by Zahra Daghighi.

\section{Financial Disclosure}

The authors declared no competing financial interests.

\section{Funding/Support}

This study was granted and supported by Pediatric In- 
fection Research Center at Mofid Hospital, affiliated to Shahid Beheshti University of Medical Sciences.

\section{References}

1. Sreeja S, Babu PRS, Prathab AG. The prevalence and the characterization of the enterococcus species from various clinical samples in a tertiary care hospital. J Clin Diagn Res. 2012;6(9):1486-8.

2. Firoozeh F, Akhi MT, Oskooei M. Antibiotic resistant in enterococcus isolated from clinical samples. J Tabriz Univ Med Sci. 2010;32(1):61-7.

3. Teymournejad O, Mohabati Mobarez A, Hosseini Doust R. Epidemiologic evaluation of Vancomycin Resistant genes in Enterococcus spp. isolated from clinical samples. J Fasa Univ Med Sci. 2011;1(2):1-6.

4. Hosseinzadeh A, Abtahi H, Shojapour M, Akbari M, Nazari R, Sofian M. Prevalence and antimicrobial susceptibility patern of vancomycin resistant enterococci spp isolated from clinical sample of educational haspitals in Arak. Arak medical university Jornal Arak Med Univ J. 2012;15(64):11-6.

5. Leclercq R, Courvalin P. Resistance to glycopeptides in enterococci. Clin Infect Dis. 1997;94:245-56.

6. Murray PR. Manual of clinical microbioloty. 6th ed. USA: Washington D.C.; 1995.
7. Viera T. Restoration of vancomycin susceptibility in Enterococcus feacalis by anti resistant determinant gene transfer. Antimicrob Chemother. 2001:973-6.

8. Cockerill F. Performance Standards for Antimicrobial Susceptibility Testing: Twenty-second Informational Supplement. Wayne PC: Clinical and Laboratory Standards Institute.; 2012.

9. Karki S, Houston L, Land G, Bass P, Kehoe R, Borrell S, et al. Prevalence and risk factors for VRE colonisation in a tertiary hospital in Melbourne, Australia: a cross sectional study. Antimicrob Resist Infect Control. 2012;1(1):31.

10. Lambiase A, Del Pezzo M, Piazza O, Petagna C, De Luca C, Rossano F. Typing of vancomycin-resistant Enterococcus faecium strains in a cohort of patients in an Italian intensive care Unit. Infection. 2007;35(6):428-33.

11. Werner $\mathrm{G}$, Klare I, Fleige $\mathrm{C}$, Witte $\mathrm{W}$. Increasing rates of vancomycin resistance among Enterococcus faecium isolated from German hospitals between 2004 and 2006 are due to wide clonal dissemination of vancomycin-resistant enterococci and horizontal spread of vanA clusters. Int J Med Microbiol. 2008;298(56):515-27.

12. Nateghian A, Robinson JL, Arjmandi K, Vosough P, Karimi A, Behzad A, et al. Epidemiology of vancomycin-resistant enterococci in children with acute lymphoblastic leukemia at two referral centers in Tehran, Iran: a descriptive study. Int J Infect Dis. 2011;15(5):e332-5. 